\title{
Antegrade perfusion during suspended animation?
}

Lars G. Svensson, MD, PhD

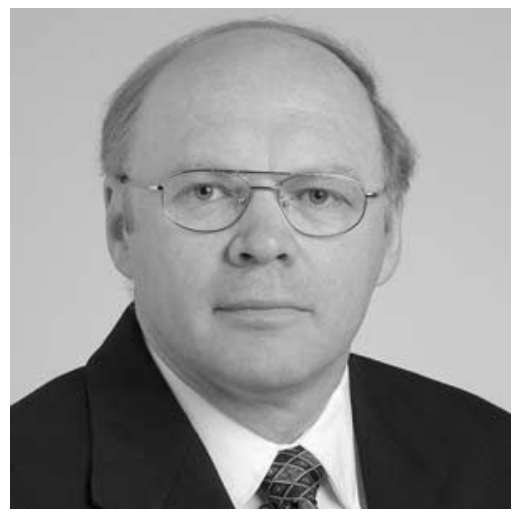

See related article on page 1080.

From the Center for Aortic Surgery and Marfan Syndrome Clinic, Department of Thoracic and Cardiovascular Surgery/F25, Cleveland Clinic Foundation, Cleveland, Ohio.

Received for publication April 26, 2002; accepted for publication May 21, 2002.

Address for reprints: Lars G. Svensson, MD, PhD, Director, Center for Aortic Surgery, Marfan Syndrome and Connective Tissue Disorder Clinic, Department of Thoracic and Cardiovascular Surgery/F25, Cleveland Clinic Foundation, Cleveland, OH 44195 (E-mail: svenssl@ccf.org).

J Thorac Cardiovasc Surg 2002;124: 1068-70

Copyright () 2002 by The American Association for Thoracic Surgery

0022-5223/2002 $\$ 35.00+0 \quad \mathbf{1 2 / 1 / 1 2 7 7 8 6}$

doi:10.1067/mtc.2002.127786

\author{
For the life of all flesh is the blood thereof. \\ -Leviticus 17:14
}

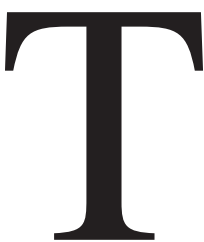

here are three important considerations when deciding whether to use antegrade brain perfusion during aortic surgery in the continuing quest to prevent, or at least significantly reduce, neurologic deficits. First, primum non nocere, do no harm. Second, how is the risk of embolism-related stroke affected? Third, what are the neurocognitive consequences?

In the early history of aortic surgery when the principle of "do no harm" was the foremost issue, the literature was replete with the high risks of strokes associated with antegrade brain perfusion. Indeed, Crawford and Saleh ${ }^{1}$ reported approximately one third of patients had strokes. They attributed this to the intimal disruption of the greater vessels from perfusion cannulas and resultant embolization of atheromatous material. Therefore, the prevailing concern of that time when using antegrade perfusion was to significantly reduce the risk of intimal damage and prevent catheter-associated embolization or dissection by using hypothermic arrest alone.

In 1995, Sabik and colleagues ${ }^{2}$ popularized the technique of using the right subclavian/axillary artery for arterial inflow for complex cardiac surgery so that a hostile aorta did not need to be cannulated. Thus, to prevent intimal damage and to be able to flush potential embolic material out of the greater vessels, my colleagues and $\mathrm{I}^{3,4}$ have recommended subclavian artery side-graft antegrade perfusion with occlusion of the innominate and left common carotid arteries with balloon catheters, without tapes encircling the arteries, and deep hypothermia with electroencephalographic brain silence. We applied this approach in our prospective randomized study $^{3,4}$ and observed that no patients had a stroke or died. The main caveat, however, is that in the situation where the subclavian artery is involved by aortic dissection, stroke may occur, probably from malperfusion of lumina or the flap acting as a flutter valve. In our study ${ }^{5}$ of 403 ascending and arch operations, the subclavian artery was dissected in 3 of our patients, and 2 of them had strokes, negating the use of this technique when dissection is found. The next question is whether it is harmful to use this technique with moderate hypothermia instead of deep hypothermia with electroencephalographic silence. As yet, the answer to this additional variable remains unknown. Whereas the perfusion pressure for antegrade perfusion has been debated, with some evidence that higher perfusion pressures may be associated with greater risk of stroke or neurocognitive deficits, currently the Stanford group ${ }^{6}$ recommends a low perfusion pressure, in agreement with the study by Di Eusanio and colleagues. ${ }^{7}$ We also favor this strategy. ${ }^{5}$

Second, addressing the problem of potential embolism-related strokes, the use of the subclavian artery for inflow has two benefits. The first is that the risk of stroke from greater vessel perfusion cannulas is reduced because, when the balloon catheters are removed, continuing the right subclavian artery perfusion flushes out potential embolic material. The other benefit is that neither the femoral artery nor the ascending aorta is cannulated, also reducing the potential source of stroke. In the study by Di Eusanio and colleagues, ${ }^{7}$ in which they did not use subclavian antegrade perfusion, $8.8 \%$ of patients had neurologic deficits and $3.7 \%$ had permanent neurologic dysfunction. In my overall experience with 427 patients undergoing ascending and/or aortic arch operations, $2.1 \%$ had neurologic deficits of which $1.2 \%$ were 
TABLE 1. Currently suggested individualized methods of brain protection in addition to brain protection protocol

\begin{tabular}{|c|c|c|c|}
\hline & \multicolumn{3}{|c|}{ ABP (RSCA) } \\
\hline & DHCA & + balloon cath & RBP (SVC) \\
\hline Acute dissection hemi-arch repair & $\sqrt{ }^{*}$ & & \\
\hline Acute dissection total arch repair & & $\sqrt{ }$ & \\
\hline Elective hemi-arch & $\sqrt{ }$ & & \\
\hline Elective hemi-arch + atheroma \pm endarterectomy & & $\sqrt{ }$ & Or $\sqrt{ }$ \\
\hline Elective total arch & & $\sqrt{ }$ & \\
\hline Elective total arch + endarterectomy & & $\sqrt{ }$ & Combined $\sqrt{ } \dagger$ \\
\hline Mini J-incision reoperations & & $\sqrt{ } \ddagger$ & \\
\hline Elephant trunk & & $\sqrt{\S}$ & \\
\hline Left thoracotomy + arch & & $\sqrt{ }$ & \\
\hline
\end{tabular}

$\overline{D H C A}$, Deep hypothermic circulatory arrest alone; $A B P(R S C A)$, antegrade brain perfusion via a side graft to a right subclavian artery and balloon occlusion of the innominate and left common arteries; $R B P(S V C)$, retrograde brain perfusion with a snare around the superior vena cava; $0 r$, either $A B P$ or RBP can be used.

*The RSCA is used for arterial inflow, especially for type I dissections, but without ABP and balloon occlusion, unless the RSCA is dissected. tCombined to maximize flushing out of embolic material and rewarming via subclavian artery to flush embolic material distally.

¥Mostly subclavian cannulation with or without circulatory arrest.

§ubclavian cannulation eliminates the need for a side graft to the aortic arch.

temporary and $0.9 \%$ permanent. Of the group of patients with antegrade perfusion via the right subclavian artery, $3.8 \%$ had neurologic deficits, either temporary or permanent, as compared with $4.1 \%$ with retrograde brain perfusion during circulatory arrest. This would suggest that there is little difference in the risk of stroke according to brain perfusion method for most patients, unless perhaps if the circulatory arrest period is prolonged.

Third, the neurocognitive consequences are more debatable and discussion is predicated by the expected duration of circulatory arrest. In our prospective randomized study of deep hypothermia and circulatory arrest with brain electroencephalographic silence in which we compared circulatory arrest alone, antegrade brain perfusion, or retrograde brain perfusion, the length of the circulatory arrest period was significantly prolonged for hemi-arch repairs using antegrade brain perfusion but not so for total arch replacements. ${ }^{3,4}$ Furthermore, patients in whom deep hypothermia and circulatory arrest alone was used more often outperformed patients receiving antegrade brain perfusion in all 9 significantly different comparisons of 51 postoperative neurocognitive tests done 3 to 6 days postoperatively, 2 to 3 weeks postoperatively, and 6 months postoperatively. ${ }^{3,4} \mathrm{On}$ the basis of these findings, our recent presentation of a study of 403 patients in whom stroke occurred in $2.0 \%, 5$ Okita's report, ${ }^{8}$ and the Mt Sinai study, ${ }^{9}$ the latter two showing potential harm with retrograde brain perfusion, I no longer use either antegrade brain perfusion or retrograde brain perfusion for the brief circulatory arrest periods required for hemi-arch repairs of 5 to 15 minutes, unless extensive atheroma is expected. However, if a total arch replacement is planned, with the concomitant expected prolonged circulatory arrest period, antegrade perfusion is mostly used; sometimes retrograde brain perfusion or a combination of the two is used in patients who are having arch and ascending aortic endarterectomies (Table 1).

Few prospective studies and even fewer randomized studies have evaluated the three methods of brain protection. Ideally, retrograde brain perfusion and antegrade brain perfusion at deep hypothermic temperatures should be compared for the longer circulatory arrest periods during total arch replacements; second, antegrade brain perfusion should be evaluated at moderate hypothermic temperatures in comparison with deep hypothermic temperatures to determine whether antegrade brain perfusion at moderate hypothermia is safe. In the study by Di Eusanio and coworkers, ${ }^{7}$ using mild or moderate hypothermia, $14.8 \%$ of patients required reoperation for bleeding, $3.7 \%$ had multiple organ failure, and $13.8 \%$ had pulmonary complications. In our series of 403 patients, ${ }^{5}$ of whom 165 were cooled to below $20^{\circ} \mathrm{C}, 3.2 \%$ required reoperations for bleeding, $8.2 \%$ had pulmonary complications, and $0.5 \%$ had multiple organ failure. From these findings, it does not appear that moderate hypothermia reduces the risk of bleeding but may in fact increase the risk because of the lack of perfusion of organs during the period of moderate hypothermic circulatory arrest, resulting in a higher incidence of multiple organ failure and bleeding complications because of poor liver protection.

In our recent study of 403 patients 5 and the older study ${ }^{10}$ of 656 patients undergoing deep hypothermia and circulatory arrest, pump time was the best predictor of neurologic deficits and also death. Other groups have also confirmed this. Unfortunately, in the study by Di Eusanio and colleagues, ${ }^{7}$ cardiopulmonary bypass time was evaluated as a categorical variable rather than a continuous variable, resulting in a loss of statistical power. This probably explains why the variable was not a significant predictor of adverse 
events. It should be emphasized that the cardiopulmonary bypass time, the cardiopulmonary bypass pump setup, technique of arterial inflow, and the medications used during the operation may in fact be more important to the outcome than the period of brief circulatory arrest and the method of brain protection used during this period of circulatory arrest. Recently, we $\mathrm{e}^{4,5}$ have described a protocol addressing parameters other than brain perfusion that we believe should further protect the brain.

Clearly, there is a role for antegrade brain perfusion for prolonged circulatory arrest periods during profound hypothermia, particularly total arch replacement. However, I caution against attempting more complex repairs using antegrade brain perfusion, such as with branch arteries and further prolonging circulatory arrest periods, particularly if moderate hypothermia is used. The most pressing reason is that this would increase bypass time and ischemia times, which in turn would lead to a greater risk for both the brain and other organs. Indeed, the risk of death increases exponentially after 65 minutes of total body circulatory arrest, apart from the effect of pump time. ${ }^{10}$

In conclusion, to protect life, newer techniques of profound hypothermic antegrade brain perfusion should be considered when the period of expected circulatory arrest will exceed approximately 30 to 40 minutes.

\section{References}

1. Crawford ES, Saleh SA. Transverse aortic arch aneurysm: improved results of treatment employing new modifications of aortic reconstruc- tion and hypothermic cerebral circulatory arrest. Ann Surg. 1981;194: 180-8.

2. Sabik JF, Lytle BW, McCarthy PM, Cosgrove DM. Axillary artery: an alternative site of arterial cannulation for patients with extensive aortic and peripheral vascular disease. J Thorac Cardiovasc Surg. 1995;109: $885-90$.

3. Svensson LG, Husain A, Penney DL, Swanson RA, Margolis DS, Kimmel WA, et al. A prospective randomized study of neurocognitive function and S-100 protein after antegrade or retrograde brain perfusion with hypothermic arrest for aortic surgery. J Thorac Cardiovasc Surg. 2000;119:163-7.

4. Svensson LG, Nadolny EM, Penney DL, Jacobson J, Kimmel WA, Entrup MH, et al. Prospective randomized neurocognitive and S-100 study of hypothermic circulatory arrest, retrograde brain perfusion, and antegrade brain perfusion for aortic arch operations. Ann Thorac Surg. 2001;71:1905-12.

5. Svensson LG, Nadolny, EM, Kimmel WA. Multimodal protocol influence on stroke and neurocognitive deficit prevention after ascending/arch aortic operations. Ann Thorac Surg. In press.

6. Frist WH, Baldwin JC, Starnes VA, Stinson EB, Oyer PE, Miller DC, et al. A reconsideration of cerebral perfusion in aortic arch replacement. Ann Thorac Surg. 1986;42:273-81.

7. Di Eusanio M, Schepens MAAM, Morschuis WJ, Di Bartolomeo R, Pierangeli A, Dossche KM. Antegrade selective cerebral perfusion during operations on the thoracic aorta; factors influencing survival and neurologic outcome in 413 patients. J Thorac Cardiovasc Surg. 2002;124:1080-6.

8. Okita Y, Minatoya K, Tagusari O, Ando M, Nagatsuka K, Kitamura S. Prospective comparative study of brain protection in total aortic arch replacement: deep hypothermic circulatory arrest with retrograde cerebral perfusion or selective perfusion. Ann Thorac Surg. 2001;72: 72-9.

9. Hagl C, Ergin MA, Galla JD, Lansman SL, McCullough JN, Spielvogel $\mathrm{D}$, et al. Neurologic outcome after ascending aorta-aortic arch operations: effect of brain protection technique in high-risk patients. $J$ Thorac Cardiovasc Surg. 2001;121:1107-21.

10. Svensson LG, Crawford ES, Hess HR, Coselli JS, Raskin S, Shenaq SA, et al. Deep hypothermia with circulatory arrest: determinants of stroke and early mortality in 656 patients. $J$ Thorac Cardiovasc Surg. 1993;106:19-31. 